\title{
THE ROLE OF GROWTH TEMPERATURE ON THE INDIUM INCORPORATION PROCESS FOR MOCVD GROWTH OF InGaN/GaN HETEROSTRUCTURES
}

\author{
Ahmad Sauffi Yusof ${ }^{1, *}$, Zainuriah Hassan ${ }^{1, *}$, Sidi Hamady ${ }^{2,3}$, Sha Shiong $\mathrm{Ng}^{1}$, Mohd Anas \\ Ahmad $^{1}$, Way Foong Lim ${ }^{1}$, Nicolas Fressengeas ${ }^{2,3}$, Christyves Chevallier ${ }^{2,3}$ \\ ${ }^{1}$ Institute of Nano Optoelectronics Research and Technology (INOR), Universiti Sains \\ Malaysia, 11800 USM, Penang, MALAYSIA. \\ ${ }^{2}$ Université de Lorraine, Laboratoire Matériaux Optiques, Photonique et Systèmes, EA 4423, \\ Metz F-57070, FRANCE. \\ ${ }^{3}$ CentraleSupélec, Laboratoire Matériaux Optiques, Photonique et Systèmes, EA 4423, Metz \\ F-57070, FRANCE.
}

*Corresponding Author: sauffi@student.usm.my,zai@usm.my

\begin{abstract}
This work presents the effect of growth temperature on the evolution of indium incorporation and growth process of $\mathrm{InGaN} / \mathrm{GaN}$ heterostructures grown using Taiyo Nippon Sanso Corporation (TNSC) metal organic chemical vapor deposition (MOCVD) SR4000-HT system. The InGaN/GaN heterostructures were epitaxially grown on $3.8 \mu \mathrm{m}$ and $40 \mathrm{~nm}$ thick undoped-GaN (ud-GaN) and $\mathrm{GaN}$ nucleation layer respectively over a commercial 2" c-plane flat sapphire substrate (FSS). The InGaN layers were grown at different temperature setting ranging from $860^{\circ} \mathrm{C}$ to $820^{\circ} \mathrm{C}$ in a step of $20^{\circ} \mathrm{C}$. The details structural, surface morphology and optical properties were investigated using X-ray diffraction (XRD), field emission scanning electron microscope (FE-SEM), atomic force microscopy (AFM) and ultraviolet-visible (UV-Vis) spectrophotometer respectively.
\end{abstract}

Keywords: InGaN, III-nitride semiconductor, MOCVD. 